\title{
Approximation of Electrical and Magneto Transport Properties of LSMO Thin Films
}

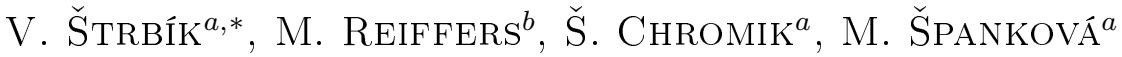 \\ ${ }^{a}$ Institute of Electrical Engineering, Slovak Academy of Sciences, Dúbravská cesta 9, 84104 Bratislava, Slovakia \\ ${ }^{b}$ Faculty of Humanities and Natural Sciences, University of Prešov, Ul. 17. novembra 1, 08116 Prešov, Slovakia
}

We have used a three term polynomial expression to approximate electrical and magneto transport properties of epitaxial $\mathrm{La}_{0.67} \mathrm{Sr}_{0.33} \mathrm{MnO}_{3}$ (LSMO) thin films in wide temperature range $(2-350 \mathrm{~K}$ ) and in magnetic field up to $9 \mathrm{~T}$. The first term is determined by a low temperature $(T<20 \mathrm{~K})$ residual resistivity $\rho_{0}$ and this term is magnetic field independent. The second term describes the electron-electron scattering in LSMO, it shows $\sim T^{2}$ dependence of resistivity up to temperature about $160 \mathrm{~K}$ and depends only weakly on magnetic field. The third term, proportional to $\sim T^{4.5}$ dependence, describes a significant increase of resistivity in temperature range 160$350 \mathrm{~K}$ and due to its sensitivity to applied magnetic field it is possible to approximate temperature dependence of magnetorestivity below $350 \mathrm{~K}$.

DOI: 10.12693/APhysPolA.126.210

PACS: 73.43.Qt, 75.47.-m, 75.70.-i

\section{Introduction}

The effort to interpret electrical and magneto transport properties of perovskite manganites in a ferromagneticmetallic $(\mathrm{FMM})$ state $\left(0<T<T_{C}, T_{M I}\right.$, where $T_{C}$ is Curie temperature and $T_{M I}$ is a temperature of metalinsulator transition) has been registered for many years. Snyder et al [1] suggested for the resistance in FMM state relation $R(T)=R_{0}+R_{2} T^{2}+R_{N} T^{N}$, where $R_{0}$, $R_{2}, R_{N}$ are fitting parameters and $N=4.5$, due to an electron-magnon scattering predicted by double exchange theory [2]. The experimental data were approximated by a polynomial fit expression for temperatures below $250 \mathrm{~K}$. Boschker et al [3] presented the polynomial fit expression in the form $\rho(T)=\rho_{0}+a_{2} T^{2}+a_{N} T^{5}$, where the term $\left(\sim T^{5}\right)$ describes the electron-phonon scattering. The polynomial fit expression approximated the resistivity data up to the temperature of $300 \mathrm{~K}$. Recently, we have introduced the polynomial fit expression in the form $\rho(T)=\rho_{0}+a_{2} T^{2}+a_{N}\left(T-T_{0}\right)^{4.5}$, where $\rho_{0}, a_{2}$, $a_{N}, T_{0}$ are fitting parameters [4]. We found out that this expression approximates well the FMM electrical transport in zero magnetic field up to a temperature of $350 \mathrm{~K}$. The first term $\rho_{0}$ represents the low temperature residual resistivity, the second term $\left(\sim T^{2}\right)$ describes the electronelectron scattering and the third term with $N=4.5$ describes the electron-magnon scattering [2]. The additional parameter $T_{0}$ indicates a temperature above which the ferromagnetic term $\left(\sim T^{4.5}\right)$ contributes to the total resistivity, whereas the low temperature $\left(T<T_{0}\right)$ resistivity is described only by the first two terms of polynomial fit expression.

In this paper we show that the magneto transport properties of $\mathrm{La}_{0.67} \mathrm{Sr}_{0.33} \mathrm{MnO}_{3}$ (LSMO) thin films can

*corresponding author; e-mail: vladimir.strbik@savba.sk be well described by the polynomial fit expression in a wide temperature range $2-350 \mathrm{~K}$ and in magnetic field at least up to $9 \mathrm{~T}$.

\section{Experimental}

The LSMO films were deposited using an on-axis dc magnetron sputtering from a stoichiometric ceramic target onto a one-side polished $\mathrm{MgO}$ (001) substrate [5]. The deposition was performed in $\operatorname{an} \operatorname{Ar}(80 \%)+\mathrm{O}_{2}(20 \%)$ atmosphere at a total pressure of $65 \mathrm{~Pa}$. The substrate was heated to $800^{\circ} \mathrm{C}$ during the deposition. The growth rate of the LSMO films was $1.2 \mathrm{~nm} / \mathrm{min}$ and the films were $100 \mathrm{~nm}$ thick. The LSMO films were subsequently in-situ annealed in $\mathrm{O}_{2}\left(10^{4} \mathrm{~Pa}\right)$ at $800^{\circ} \mathrm{C}$ for 1 hour.

The magnetotransport properties were measured in magnetic field $0,4.5$, and $9 \mathrm{~T}$ oriented parallel to the film surface, in a temperature range $2-400 \mathrm{~K}$ using a four-probe method with physical properties measurement system (PPMS). The patterning of the basic structure $\left(10 \times 10 \mu \mathrm{m}^{2}\right)$ was carried out by a photolithography process and argon ion beam etching with cooling of the substrate.

\section{Results and discussion}

The magnetic properties (Curie temperature $>400 \mathrm{~K}$ ) and structural characterization of the epitaxial LSMO films with enhanced temperature of metal-insulator transition $T_{M I}=406 \mathrm{~K}$ were described elsewhere [5]. The experimental resistivity vs. temperature $(\rho-T)$ dependences in magnetic field of $0,4.5$ and $9 \mathrm{~T}$ are shown in Fig. 1 (points). These dependences were approximated by the polynomial fit expression $\rho(T)=\rho_{0}+a_{2} T^{2}+$ $a_{N}\left(T-T_{0}\right)^{4.5}$ (Fig. 1, full lines). The average squared errors of fitting curves are $2.43 \times 10^{-3}, 6.34 \times 10^{-4}$ and $2.41 \times 10^{-4}$ for curves at $0,4.5$ and $9 \mathrm{~T}$, respectively. Very good agreement was achieved in temperature range 2-350 $\mathrm{K}$ for $B=0 \mathrm{~T}$, at magnetic field of $9 \mathrm{~T}$ even up to $380 \mathrm{~K}$. In case of increasing magnetic field the experimental curves more and more approach the magnetic 


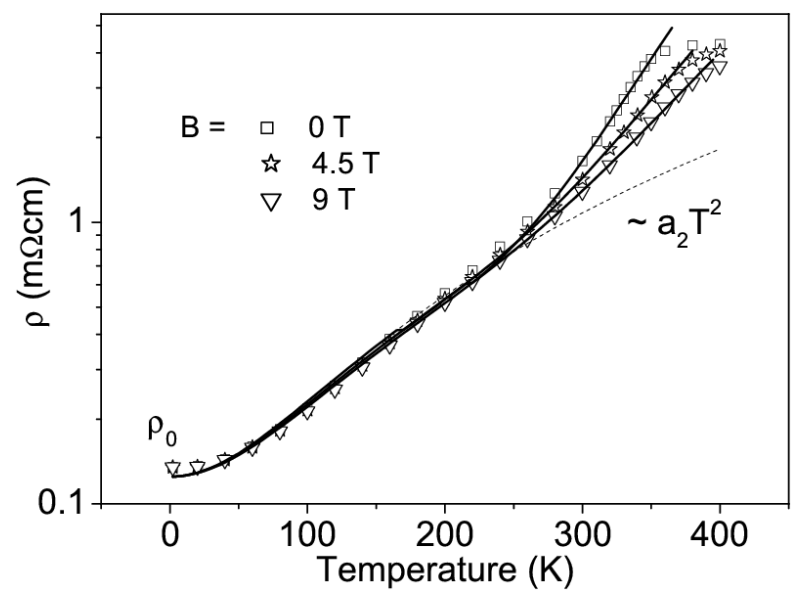

Fig. 1. Approximation (full line) of $\rho-T$ dependence of the LSMO film in magnetic field $0,4.5$ and $9 \mathrm{~T}$ (points). Dashed line represents the sum of first two terms of expression.

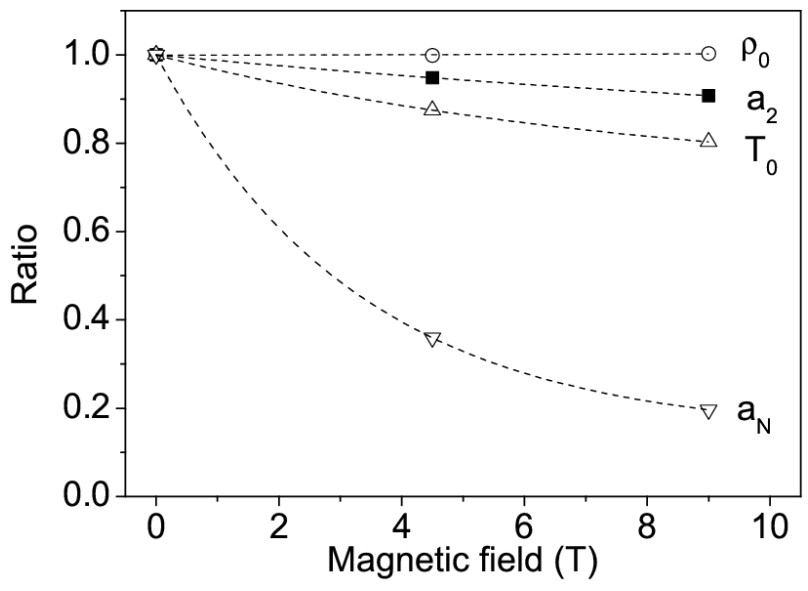

Fig. 2. Magnetic field dependences of fitting parameters $\rho_{0}, a_{2}, T_{0}$ and $a_{N}$.

field independent behaviour of the first two terms $\rho_{0}$ and $a_{2} T^{2}$ (dashed line in Fig. 1).

The magnetic field dependence of the fitting parameters is shown in Fig. 2. Whereas the parameter $\rho_{0}$ is magnetic field independent, the second term depends on the magnetic field only weakly $(<10 \%)$. The magnetic field has a significant influence on fit parameters of the third term $T_{0}$ and $a_{N}$. $T_{0}$ parameter decreases by $20 \%$ at $B=9 \mathrm{~T}, a_{N}$ parameter decreases by as much as $80 \%(5$ times smaller than at $B=0 \mathrm{~T})$. It means that the magneto transport properties of LSMO are primarily concentrated in the third (ferromagnetic) term.

Two temperature dependences of the magnetoresistivity for two values of magnetic field 4.5 and $9 \mathrm{~T}$ are shown in the Fig. 3. The magnetoresitivity is defined as $M R=[\rho(B)-\rho(B=0)] / \rho(B=0)$. The experimental magnetoresistivities (points) are well approximated by the one computed from the fitting curves (full lines). Our results (Figs. 1 and 3) show that the upper temper-

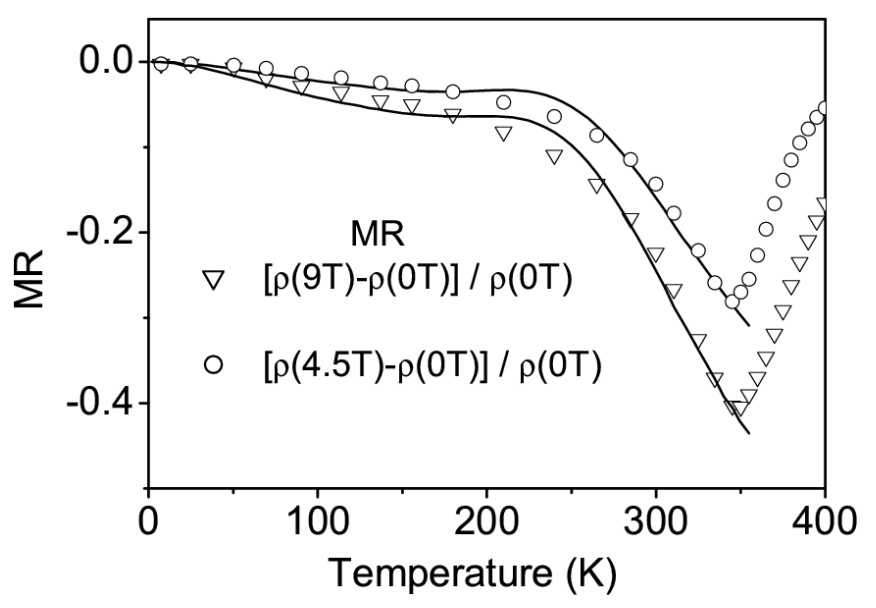

Fig. 3. Experimental (points) and approximated (full line) magnetoresistivity for two values of magnetic field.

ature limit of the three term polynomial approximation is about $350 \mathrm{~K}$ for the LSMO thin films with enhanced $\mathrm{T}_{M I}$ values.

\section{Conclusions}

The three term polynomial expression $\rho(T)=\rho_{0}+$ $a_{2} T^{2}+a_{N}\left(T-T_{0}\right)^{4.5}$ was used to describe the properties of the epitaxial LSMO thin films under magnetic field. The first term (residual resistivity $\rho_{0}$ ) is temperature and magnetic field independent. The second term characterizes the electron-electron scattering and it depends on magnetic field only weakly. The third term plays a crucial role to interpret the ferromagnetic properties of manganites. Its temperature dependence $\sim T^{4.5}$ is in agreement with the double exchange theory and its sensitivity to applied magnetic field was used to approximate the magnetoresistivity dependence on the temperature. The polynomial fit expression well describes the electrical and magneto transport properties of the LSMO in a wide temperature range $(2-350 \mathrm{~K})$ and in magnetic field at least up to $9 \mathrm{~T}$.

\section{Acknowledgments}

This work was supported by the Slovak Grant Agency VEGA under projects Nos. 2/0173/13, 2/0164/11, 2/0070/12 and APVV-0494-11.

\section{References}

[1] G.J. Snyder, R. Hiskes, S. DiCarolis, M.R. Beasley, T.H. Geballe, Phys. Rev. B 53, 14434 (1996).

[2] K. Kubo, N. Ohata, J. Phys. Soc. Jpn 33, 21 (1972).

[3] H. Boschker, M. Huijben, A. Vailions, J. Verbeeck, S. van Aert, M. Luysberg, S. Bals, G. van Tendeloo, E.P. Houwman, G. Koster, D.H.A. Blank, G.J. Rijnders, Phys. D: Appl. Phys. 44, 205001 (2011).

[4] V. Štrbík, Š. Chromik, J. of Eletrical Engineering 63, 270 (2012).

[5] Š. Chromik, V. Štrbík, E. Dobročka, A. Dujavová, M. Reiffers, J. Liday, M. Španková, Appl. Surf. Sci 269, 98 (2013). 